\title{
Estudio Preliminar del Material Particulado de Fuentes Estacionarias: Aplicación al Sistema de Compensación de Emisiones en la Región Metropolitana, Chile
}

\author{
Margarita Préndez $^{(1)}$, Roberto M. Corvalán ${ }^{(2)}$ y Michael Cisternas ${ }^{(3)}$ \\ (1) Universidad de Chile, Facultad de Ciencias Químicas y Farmacéuticas, Depto. de Química \\ Orgánica y Fisicoquímica, Olivos 1007, Casilla 233, Santiago-Chile (e-mail: mprendez@ciq.uchile.cl) \\ (2) Universidad de Chile, Facultad de Ciencias Físicas y Matemáticas, Depto. de Ingeniería \\ Mecánica, Casilla 2777, Santiago-Chile (e-mail: rocorval@cec.uchile.cl) \\ (3) Mutual de Seguridad, Chile. Av. B. O’Higgins 194, Santiago-Chile \\ (e-mail: mcisternas@mutualseg.cl)
}

\begin{abstract}
Resumen
Se realiza una caracterización física y química de emisiones proveniente de diferentes tipos de fuentes estacionarias en Santiago de Chile con el propósito de determinar si la forma en que se aplican las medidas de compensación de emisiones es apropiada. El sistema de compensación de emisiones se aplica tradicionalmente a las emisiones másicas. La hipótesis de este trabajo es que el daño a la salud ocasionado por el material particulado depende además de su tamaño y de los elementos químicos que contenga, como lo corroboran los resultados que muestran notables diferencias en la composición química de las emisiones desde distintos tipos de fuentes. Esto indica que la aplicación del sistema actual no es apropiada como herramienta ambiental para el mejoramiento de la calidad del aire de la ciudad.
\end{abstract}

Palabras claves: fuentes estacionarias, compensación de emisiones, caracterización del particulado

\section{Preliminary Study of Particulate Matter from Stationary Sources: Application to the Emission Compensation System of the Metropolitan Region, Chile}

\begin{abstract}
A physico-chemical characterization of emissions from different types of stationary sources in Santiago-Chile was carried out with the purpose of determining if the form in which environmental management policies is well applied. The emission compensation system is currently applied on the basis of total mass emissions. The hypothesis of this work is that damage to health from particulate matter exposure also depends strongly on particulate size and chemical elements as is confirm by the results showing notable differences in chemical composition of the emissions from different source types. This indicates that the application of the current emission compensation system is not an environmental tool appropriated for improving the quality of the air in the city.
\end{abstract}

Keywords: stationary source emissions, emission compensation program; particulate matter 


\section{INTRODUCCIÓN}

Santiago está situado en una cuenca cuyas condiciones geográficas y climatológicas favorecen los altos niveles de contaminación que la caracterizan, debido a la falta de ventilación. Sostenidos aumentos en la concentración de material particulado respirable obligó a las autoridades locales a diseñar estrategias de gestión ambiental pública para reducir o al menos detener el aumento de las emisiones en la ciudad.

Una de dichas estrategias es la implementación de un sistema de compensación de emisiones a través del cual se asigna a fuentes estacionares existentes en la ciudad, una especie de "derecho" para cierta cantidad de emisiones. Toda nueva fuente que inicie operaciones en la región, debe compensar sus emisiones a través de la reducción de emisiones de una o más fuentes (MINSAL, 1992). Esta medida se basó en la cuantificación de las emisiones másicas totales, lo que en la práctica, permite compensar entre emisiones provenientes de diferentes fuentes con, asimismo, un diferente potencial de impacto en salud, debido a las diferencias en tamaño y composición química que dichas emisiones puedan presentar. Esto contradice los resultados de investigaciones a nivel internacional que aún están orientadas al estudio de diferentes parámetros que influyen sobre el impacto en salud por exposición a material particulado, como por ejemplo la distribución de tamaño en masa y/o número, la composición química, el área superficial de las partículas y otros (Ntziachristos y Samara, 2003).

Debido a que la química asociada a la contaminación atmosférica es de alta complejidad, los efectos de las emisiones desde fuentes estacionarias sobre la química atmosférica resultante no son suficientemente conocidos.

Es posible, en consecuencia, que estrategias de control bien intencionadas den como resultado un aumento de la toxicidad del aire contaminado (Phalen, 2004).

En este contexto se realiza un estudio orientado a la caracterización de la distribución de tamaño en número de partículas y composición elemental de las emisiones de material particulado provenientes de una muestra de fuentes estacionarias de diverso tipo que cumplen en todo momento con los estándares vigentes de emisión en masa.

\section{ANTECEDENTES}

\section{Características del Material Particulado Respirable}

El material particulado (PM) es un contaminante de naturaleza compleja no sólo por sus características físicas (masa, tamaño, y densidad), sino también por sus características químicas (contiene compuestos orgánicos e inorgánicos, metales y contaminantes primarios y secundarios). Estas caracteresticas son críticas para determinar el tipo y magnitud de los efectos sobre la salud humana.

Las partículas suspendidas presentes en la atmósfera tienen un rango de tamaño entre 0,001 y $50 \mu \mathrm{m}$ (Morawska et al., 1999). La fracción de aerosoles respirables con diámetro aerodinámico menor o igual a $10 \mu \mathrm{m}$ (PM10), penetran a diferentes profundidades del sistema respiratorio. Las partículas más pequeñas son respirables en mayor portentaje. Las partículas con diámetro aerodinámico igual o menor a $2,5 \mu \mathrm{m}$ son $100 \%$ respirables (PM2,5). Las partículas más pequeñas de $0,5 \mu \mathrm{m}$ pueden penetrar los mecanismos de defensa del sistema respiratorio y depositarse en los alvéolos pulmonares (Préndez, 1993; Cohen y Hering, 1995).

El comportamiento de las partículas en la atmósfera está determinado por su tamaño, siendo las de menor tamaño afectadas por movimientos de tipo browniano y las de mayor tamaño por fuerzas gravitacionales e inerciales (Lazar et al., 1999).

Los tiempos de residencia de las partículas en la atmósfera, también dependen de su tamaño. Partículas de tamaño menor a 2,5 $\mu \mathrm{m}$ pueden tener tiempos de residencia de días 0 incluso semanas y podrían ser transportadas miles de kilómetros (Sosaky et al., 1988). Partículas de tamaño mayor a 2,5 $\mu \mathrm{m}$ tienen tiempos de residencia de minutos a horas y pueden ser transportadas hasta unos pocos centenares de kilómetros (Vana et al., 1999). En la fracción más fina $(<2,5 \mu \mathrm{m})$, se encuentran los sulfatos y los nitratos los cuales presentan potencial de daño a la salud y causan una disminución en la visibilidad (Baik et al., 1996). 
Adicionalmente, se encuentran compuestos orgánicos como los hidrocarburos aromáticos policíclicos, algunos con potencial cancerígeno y mutagénico (Sienra et al., 2005). También es posible encontrar metales pesados como $\mathrm{Pb}, \mathrm{Cd}, \mathrm{Ni}, \mathrm{Cu}, \mathrm{Mo}$ y $\mathrm{V}$, que tienen variados efectos sobre la salud humana (Préndez et al, 1991; Goyer, 1995; Ondov y Wexler, 1998; WHO, 2000). Estas partículas son esencialmente de origen antropogénico, asociado a procesos de combustión y a varios procesos productivos relacionados con la presencia de compuestos orgánicos y especies inorgánicas (Hosiokangas et al, 1999; Sudesh y Rajamani, 2006). Algunos de éstos son elementos esenciales para el crecimiento, reproducción y supervivencia de organismos vivos, pero otros poseen un elevado potencial de daño para la salud humana y el ambiente aún en bajas concentraciones. Muchos de los efectos tóxicos pueden asociarse con poblaciones discretas de aerosoles desde fuentes individuales o tipos de fuentes genéricas (Ondov y Wexler, 1998).

Metales de transición asociados con PM10, que pueden generar radicales hidroxilos produciendo daño oxidativo, pueden ser especialmente peligrosos (Donaldson et al., 1998); sin embargo, la respuesta provocada por la producción y liberación de mediadores inflamatorios de las vías respiratorias parece deberse a metales específicos (Carter et al., 1997). Por otra parte, Costa y Dreher (1997) indican que la dosis de metales biodisponibles, y no la masa de partículas, se relaciona estrechamente con lesiones asociadas con el sistema cardiopulmonar.

La US EPA (1999) define a Be y $\mathrm{Hg}$ como elementos altamente peligrosos; elementos como $\mathrm{Ba}, \mathrm{Cd}, \mathrm{Cu}, \mathrm{Mn}, \mathrm{V}$ y $\mathrm{Sn}$ han sido también identificados como elementos con alto potencial de peligrosidad. Todos estos elementos, a excepción del $\mathrm{Mn}$, han sido identificados como elementos traza y todos estos a excepción del $\mathrm{Ba}$, son metales pesados (Duffus, 1983), además de ser emitidos por diferentes tipos de fuentes estacionarias de combustión (Mukherjee et al, 2003).

Perspectiva de las Fuentes Estacionarias en la Región Metropolitana de Santiago, Chile

De acuerdo con la información oficial disponible, hay algo más de 5000 fuentes estacio- narias en la Región Metropolitana de Santiago. El $82 \%$ de éstas están documentadas con información completa que permite su caracterización. Estas fuentes pueden ser clasificadas en siete grandes grupos: calderas industriales, calderas de calefacción, calderas de fluído térmico, generadores de vapor, hornos industriales, hornos de panadería y procesos industriales.

De acuerdo con el registro de fuentes en las bases de datos administradas por la Autoridad Sanitaria dependiente del Ministerio de Salud, las calderas de calefacción representan un $40,3 \%$ del total de fuentes; los hornos de panaderías un 25\%; los generadores de vapor un 13,8\%; los procesos industriales un 10,9\%; los hornos industriales un $7,9 \%$; las calderas de fluido térmico un $1,2 \%$ y las calderas industriales un $0,9 \%$.

Dependiendo del caudal volumétrico de los gases de escape, las fuentes estacionarias se clasifican en puntuales (caudal $\geq 1000 \mathrm{~m}^{3} \mathrm{~h}^{-1}$ ) y grupales (caudal $<1000 \mathrm{~m}^{3} \mathrm{~h}^{-1}$ ). El Decreto Supremo $N^{\circ}$ 4/1992 (MINSAL, 1992) de aplicación en la Región Metropolitana, establece las normas de emisión de material particulado a fuentes estacionarias. Estos estándares son $56 \mathrm{mgNm}^{-3}$ a partir del 31 de diciembre de 1997; $50 \mathrm{mgNm}^{-3}$ a partir del 31 de diciembre de 1999 y $32 \mathrm{mgNm}^{-3}$ a partir del 31 de diciembre del 2004. De acuerdo a la última información disponible, 342 fuentes, correspondientes al $7 \%$ del total de fuentes registradas, tienen emisiones mayores que 32 $\mathrm{mgNm}^{-3}$ y deberían reducir o compensar emisiones con el propósito de cumplir el estándar de emisión.

\section{El Sistema de Compensación de Emisiones}

El Plan de Prevención y Descontaminación Atmosférica de la Región Metropolitana de Santiago (PPDA), es un instrumento de gestión ambiental pública que se aplica desde 1997. Este plan incorpora un sistema de compensación de emisiones como estrategia para reducir las emisiones de material particulado, sin entorpecer el crecimiento industrial de la región.

La compensación se entiende como un acuerdo entre titulares de fuentes, en el sentido que si se verifica una reducción de emisiones en una fuente dada, esto permitiría la incorporación de una fuente nueva o bien la 
expansión de otra existente. De acuerdo con lo establecido por el sistema de compensación de emisiones (http://www.conama.cl /portal/1255/articles-25999_ppda.pdf), toda nueva fuente que iniciara actividades en la región, a partir del 2004 debía compensar el $150 \%$ de sus emisiones estimadas. Adicionalmente, dependiendo de la magnitud de sus emisiones en relación con una cuota individual definida para fines del 2004, las nuevas fuentes que reemplazan fuentes existentes deben compensar un $100 \%$ de las emisiones correspondientes a ese año, si las emisiones de la nueva fuente son iguales 0 menores que la cuota de emisión y el $150 \%$ de la fracción de emisión que excede esta cuota. De esta forma las fuentes estacionarias puntuales tienen definido un límite de emisión diaria, que se puede calcular mediante la siguiente expresión:

$E_{D}=Q \times C \times H$

donde $E_{D}$ es la emisión diaria en $\mathrm{Kg}$ día $^{-1} ; Q$ es el caudal de gases de escape en $\mathrm{m}^{3} \mathrm{~h}^{-1} ; \mathrm{C}$ es la concentración másica de contaminante en $\mathrm{Kg} \mathrm{m}^{-3}$ y $\mathrm{H}$ son las horas de operación al día

El sistema se basa en las emisiones másicas totales, lo que implica que no se hace distinción alguna por tamaño de partículas o composición química. La hipótesis planteada en esta investigación es que, dependiendo del tipo de fuente emisora y el proceso que tiene lugar, existe un amplio rango de tamaños de partícula y elementos químicos presentes y, en consecuencia es razonable esperar que las emisiones desde distintos tipos de fuentes sean responsables también de diferentes efectos en salud, tanto en naturaleza como en magnitud.

\section{METODOLOGÍA}

\section{Selección de la Muestra}

La Autoridad Sanitaria, dependiente del Ministerio de Salud, es la institución responsable de la fiscalización de las emisiones desde fuentes estacionarias, utilizando infraestructura propia o bien laboratorios externos debidamente autorizados. La fiscalización se realiza a través de la aplicación del método de medición US EPA CH-N5. La utilización de este método considera la toma de muestra a través de filtros de fibra de vidrio, los que luego se pueden utilizar para análisis de laboratorio.

De acuerdo a las regulaciones ambientales vigentes, cada fuente se debe someter a un control de emisiones, al menos una vez al año, y los laboratorios deben conservar los filtros al menos durante cuatro meses.

Para seleccionar la muestra de filtros a analizar, se intentó, en primer término, cubrir todos los tipos de procesos presentes en la ciudad señalados anteriormente, para lo cual se agruparon por tipo de proceso, área de producción y tipo potencial de emisiones asociadas a estos procesos.

Debido al corto período de tiempo en que los laboratorios están obligados a conservar las muestras, el uso de métodos alternativos al US EPA $\mathrm{N}^{\circ} 5$ y la incerteza sobre las condiciones de conservación de algunas muestras, el universo de filtros disponibles para seleccionar se redujo drásticamente. De esta forma, si bien la muestra puede no ser representativa del número de fuentes presentes en la ciudad, en ella están representados la mayoría de los principales procesos industriales, siendo posible entonces, analizar variaciones en la distribución de tamaño y la composición elemental del material particulado emitido por estos procesos. De esta forma, se seleccionaron filtros provenientes de los siguientes tipos de fuentes: hornos industriales (horno de de cocción, horno Morgan, horno de quemado, horno de fundición de silicatos y horno de polimerización), procesos industriales (molino, pulidora, secador spray y granalladora), generador de vapor y caldera industrial,

En algunos tipos de fuentes fue posible seleccionar dos filtros para analizar eventuales variaciones temporales tanto de la composición elemental como de las distribuciones de tamaño de partícula (horno de fundición de silicatos, horno Morgan, granalladora, pulidora y calderas industriales), haciendo un total de 17 filtros ( 7 filtros de hornos industriales, 7 de procesos industriales, 2 de calderas industriales y 1 de generador de vapor). Cada filtro se dividió en cuatro partes; una fracción se utilizó para identificar y cuantificar elementos químicos, otra se utilizó para determinar la distribución de tamaño de partículas y las dos fracciones restantes se dejaron como contramuestras. 


\section{Técnicas Analíticas}

Para la determinación de la distribución por tamaño de partículas se utilizó un instrumento Masterziser $X$, de Malvern Instruments posterior a una extracción acuosa del material particulado. Esta medición se basa en el método LALS (low angle light scattering), a través de un láser $\mathrm{He}-\mathrm{Ne}$ que opera como fuente de luz de $633 \mathrm{~nm}$ de longitud de onda, un sistema óptico y un detector fotosensible que permite configurar un patrón de difracción que es analizado y calculado a través del software incorporado al equipo.

Para la cuantificación de elementos contenidos en los filtros se utilizaron las técnicas analíticas de Espectroscopía de emisión óptica con plasma inductivamente acoplado (ICP-OES) y la Espectrometría de masa con plasma inductivamente acoplado (ICP-MS). Se utilizó una adaptación del método EPA 6010B para la digestión ácida en un horno micro-ondas. Dado que los filtros de fibra de vidrio tienen mayores valores en blanco que otros filtros, la digestión en cada filtro consideró la muestra, los blancos de reactivos y los blancos obtenidos de la parte no impactada del filtro; los valores finales informados corresponden a las concentraciones netas. Los límites de detección para ambas técnicas ICP son los que se indican en la Tabla 1.

Como métodos de validación se efectuaron pruebas de confiabilidad, interferencia e intercomparación de técnicas, como parámetros de control de calidad. La exactitud se determinó utilizando un material certificado de alta pureza $N^{\circ}$ DWPS. Para el control de interferencias se siguió el criterio general contenido en el método EPA 6010B.

En la Tabla 2 se muestra la intercomparación entre las técnicas ICP-OES e ICP-MS para tres muestras. Los resultados evidencian que la técnica ICP-MS es más sensible para algunos elementos y tiene límites de detección para elementos como Be, V, Mn, Co y Sb,que no son o no pueden ser determinados por la técnica ICP-OES. Para Mg, Al, Cu, Zn, As y $\mathrm{Pb}$, cuyas concentraciones en las tres muestras están sobre los límites de detección, se puede estimar la incertidumbre asumiendo una regresión lineal entre los valores de las dos técnicas; sus pendientes interceptan los errores estándar a un nivel de confianza de $95 \%$.
Tabla 1: Límites de detección de elementos para las técnicas ICP-OES y ICP-MS

\begin{tabular}{crr}
\hline \multirow{2}{*}{ ELEMENTOS } & \multicolumn{2}{c}{ TECNICAS } \\
\cline { 2 - 3 } & $\begin{array}{r}\text { ICP-OES } \\
\left(\mu \mathrm{g} \mathrm{L}^{-1}\right)\end{array}$ & $\begin{array}{r}\text { ICP-MS } \\
\left(\mu \mathrm{g} \mathrm{L}^{-1}\right)\end{array}$ \\
\hline $\mathrm{Be}$ & $\mathrm{ND}$ & 4,0 \\
$\mathrm{Mg}$ & $\mathrm{ND}$ & 10,0 \\
$\mathrm{Al}$ & 7,8 & 20,0 \\
$\mathrm{~V}$ & $\mathrm{ND}$ & 4,0 \\
$\mathrm{Cr}$ & 2,0 & 4,0 \\
$\mathrm{Mn}$ & $\mathrm{ND}$ & 2,0 \\
$\mathrm{Fe}$ & 2,5 & -- \\
$\mathrm{Co}$ & $\mathrm{ND}$ & 2,0 \\
$\mathrm{Ni}$ & 2,6 & 4,0 \\
$\mathrm{Cu}$ & 1,4 & 4,0 \\
$\mathrm{Zn}$ & 1,4 & 20,0 \\
$\mathrm{As}$ & 6,4 & 8,0 \\
$\mathrm{Se}$ & 5,0 & 8,0 \\
$\mathrm{Mo}$ & $\mathrm{ND}$ & 4,0 \\
$\mathrm{Cd}$ & 0,67 & 2,0 \\
$\mathrm{Sb}$ & $\mathrm{ND}$ & 2,0 \\
$\mathrm{~Pb}$ & 8,0 & 4,0 \\
\hline ND: No determinado & \\
\hline
\end{tabular}

Tabla 2: Intercomparación de técnicas ICP. Valores en $\mathrm{mg} \mathrm{L}^{-1}$

\begin{tabular}{|c|c|c|c|c|c|c|}
\hline \multirow{2}{*}{$\begin{array}{l}\text { Ele- } \\
\text { mento }\end{array}$} & \multicolumn{2}{|c|}{ MC1 } & \multicolumn{2}{|c|}{ MC2 } & \multicolumn{2}{|c|}{ MCB } \\
\hline & MS & OES & MS & OES & MS & OES \\
\hline $\mathrm{Be}$ & 0,01 & ND & 0,01 & ND & 0,01 & ND \\
\hline $\mathrm{Mg}$ & 11 & 12 & 109 & 98,3 & 166 & 156 \\
\hline Al & 36 & 41 & 556 & 521 & 524 & 529 \\
\hline V & 0,1 & ND & 0,4 & 0,2 & 0,1 & ND \\
\hline $\mathrm{Cr}$ & 0,1 & BLD & 0,5 & 0,04 & 0,1 & BLD \\
\hline $\mathrm{Mn}$ & 0,2 & $N D$ & 0,3 & 0,2 & 0,2 & 0,3 \\
\hline Co & 0,01 & ND & 0,03 & ND & 0,02 & ND \\
\hline $\mathrm{Ni}$ & 0,1 & 0,07 & 0,2 & BLD & 0,1 & BLD \\
\hline $\mathrm{Cu}$ & 8,6 & 9,2 & 12 & 16 & 0,2 & 0,1 \\
\hline $\mathrm{Zn}$ & 52 & 47 & 10 & 8,8 & 1,1 & 0,8 \\
\hline As & 0,1 & 0,10 & 0,3 & 0,1 & 14 & 12 \\
\hline $\mathrm{Se}$ & 0,1 & BLD & 0,3 & BLD & 0,2 & 0,0 \\
\hline Mo & 0,01 & ND & 0,1 & ND & 0,02 & ND \\
\hline $\mathrm{Cd}$ & 0,4 & 0,4 & 0,3 & BLD & 0,01 & 0,0 \\
\hline $\mathrm{Sb}$ & 0,1 & ND & 0,1 & ND & 0,3 & 0,2 \\
\hline $\mathrm{Pb}$ & 19 & 17 & 25 & 20 & 0,3 & 0,4 \\
\hline
\end{tabular}

BLD: bajo del límite de detección

ND: no determinado

De esta forma se obtiene que la técnica ICP. MS entrega mayores valores que la técnica de ICP-OES para: $\mathrm{Mg}$ en un $8 \pm 4 \%$, para $\mathrm{Al}$ en un $4 \pm 7 \%$, para $Z n$ en un $10,1 \pm 0,2 \%$, para As en un $16 \pm 1,5 \%$ y para $\mathrm{Pb}$ en un $21,5 \pm 11,3 \%$. Sin embargo, en el caso del $\mathrm{Cu}$ la técnica ICP-OES entrega el valor mayor en un $29,7 \pm 20,5 \%$. Considerando estos 
resultados, se decidió utilizar los valores entregados por la técnica IPC-MS, excepto en el caso de $\mathrm{Fe}$, para el cual se utilizaron los resultados obtenidos mediante ICP-OES, dado que la técnica ICP-MS no es adecuada para la cuantificación de dicho elemento.

\section{RESULTADOS}

\section{Distribución por Tamaño de Partícula}

Las Figuras 1 y 2 muestran la distribución porcentual de partículas versus el diámetro de partícula para los 17 filtros analizados. Los resultados evidencian que la mayor parte de las partículas son respirables (tamaño menor que $10 \mu \mathrm{m})$. Un análisis más detallado muestra que un $96 \%$ de ellas tiene un tamaño menor que $9,9 \mu \mathrm{m}$, un $88,5 \%$ tiene tamaño menor que $2,3 \mu \mathrm{m}$ y un $66,6 \%$ tiene un tamaño en el rango de 0,05 a $1 \mu \mathrm{m}$. Además, se detectaron dos máximos relativos para diferentes tipos de fuentes siendo posible definir los dos grupos de fuentes indicados en las Figuras 1 y 2 . En efecto, la Figura 1 muestra un primer máximo en el rango de 0,28 a $0,43 \mu \mathrm{m}$ y un segundo máximo en el rango de 4,3 a $8,04 \mu \mathrm{m}$.

En la Figura 2 se aprecian máximos relativos en el rango de 0,53 a $1,0 \mu \mathrm{m}$ y en $8 \mu \mathrm{m}$, respectivamente. Descartando el máximo correspondiente a 1,2 $\mu \mathrm{m}$, el rango de tamaño de partículas donde se verifica el primer máximo relativo disminuye de 0,53 a 0,81 $\mu \mathrm{m}$, considerando todo el resto de filtros.

\section{Análisis Químico}

Se determinaron las concentraciones de elementos químicos a través de las técnicas antes señaladas y haciendo uso de la información adicional contenida en las bases de datos que administra la Autoridad Sanitaria para fines de fiscalización. En estas bases de datos se registra la masa $(M)$ de cada filtro, el caudal de gases de escape $(Q)$, las concentraciones totales de material particulado $\left(\mathrm{C}_{\mathrm{PM}}\right)$ y las horas diarias $(\mathrm{H})$ de operación de la fuente.

Aplicando la ecuación (1), se calculó la emisión másica diaria de cada elemento y la emisión másica total de material particulado. Sin embargo, no todas las fuentes analizadas tenían la información completa, por lo que la concentración elemental y la emisión másica de particulado sólo se pudo calcular para seis de los filtros recolectados (caldera industrial, horno de polimerización, horno Morgan, secador molino y pulidora).

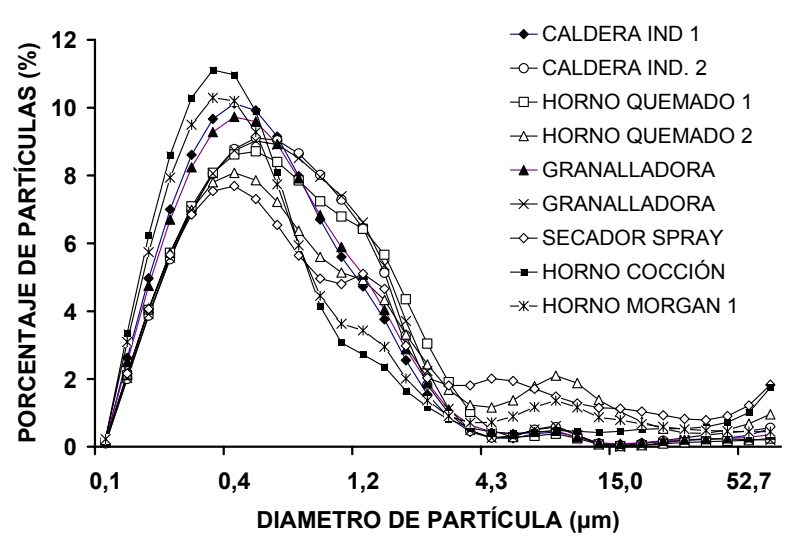

Fig. 1. Distribución porcentual de número de partículas versus diámetro para rango de tamaños de partícula máximos de $0,28 \mu \mathrm{m}-0,43 \mu \mathrm{m}$

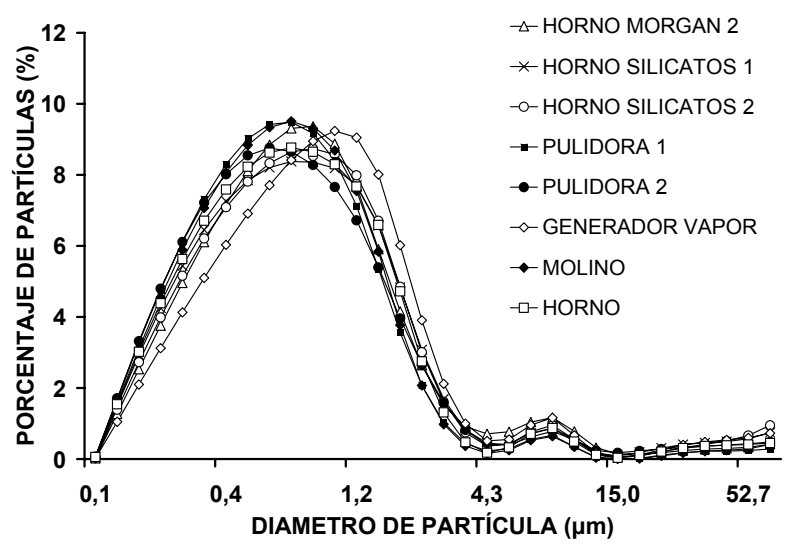

Fig. 2. Distribución porcentual de número de partículas versus diámetro para rango de tamaños de partícula máximo de $0,53 \mu \mathrm{m}-1,00 \mu \mathrm{m}$

En la Tabla 3 se muestran los resultados obtenidos para la cuantificación de los elementos químicos correspondientes a los filtros que cuentan con información completa. Los resultados muestran que diferentes tipos de fuentes emiten diferentes elementos en concentraciones que les son propias. Entre los elementos detectados se encuentran aquéllos declarados por la US EPA (1999) como los más peligrosos para la salud, como son $\mathrm{Cr}, \mathrm{Mn}, \mathrm{Ni}, \mathrm{Pb}$ y $\mathrm{Cd}$. La Organización Mundial de la Salud (OMS), también considera como elementos dañinos para la salud a $\mathrm{Cd}, \mathrm{Pb}, \mathrm{Mn}, \mathrm{Hg}, \mathrm{Pt}$ y $\vee$ (efectos sobre cáncer) y $\mathrm{As}, \mathrm{Cr}(\mathrm{VI})$ y $\mathrm{Ni}$ (peligro de contraer cáncer).

Adicionalmente, dos muestras provenientes de una misma fuente (Calderas 1 y 2 ) mues-

Información Tecnológica - Vol. 18 Nº 2 - 2007 
tran diferencias en los elementos químicos detectados. En efecto, se detectaron $\mathrm{Cr}, \mathrm{Mn}$, $\mathrm{Ni}$ y $\mathrm{Pb}$ en las emisiones de ambas calderas y $\mathrm{Mg}$, Fe y $\mathrm{Cu}$ adicionalmente en las emisiones de la caldera 2. Aplicando la ecuación (1), se obtienen las siguientes emisiones másicas diarias $E_{D 1}=1,01 \mathrm{Kg}$ día ${ }^{-1}$ para la caldera $1 \mathrm{y}$ $E_{D 2}=0,18 \mathrm{Kg} \mathrm{dia}^{-1}$ para la caldera 2 .

Usando las concentraciones elementales obtenidas con los análisis químicos (Tabla 3), se calcularon las emisiones másicas diarias ( $\mathrm{g}$ día $^{-1}$ ) de los elementos químicos según se muestra en la Figura 3, para ambas calderas. Se observa que, a pesar de la menor emisión másica diaria total de PM de la caldera 2 en comparación con la 1 , ella produce mayores cantidades de $\mathrm{Cr}$, Mn, $\mathrm{Ni}$ y $\mathrm{Pb}$.

Tabla 3: Concentración de elementos Quím.-cos emitidos por fuentes estacionarias. Valores en $\mathrm{mg} \mathrm{m}^{-3} \mathrm{~N}$

\begin{tabular}{|c|c|c|c|c|c|c|c|}
\hline 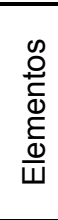 & 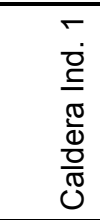 & 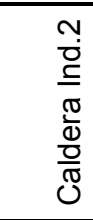 & 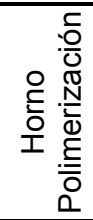 & 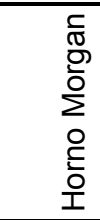 & 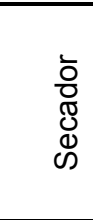 & $\frac{\stackrel{\circ}{\leftrightarrows}}{\stackrel{0}{\frac{0}{2}}}$ & $\begin{array}{l}\frac{\pi}{0} \\
\frac{0}{3} \\
\frac{0}{2}\end{array}$ \\
\hline $\mathrm{Mg}$ & BLD & 1,244 & BLD & BLD & BLD & 2,446 & BLD \\
\hline V & BLD & BLD & BLD & 0.026 & BLD & BLD & BLD \\
\hline $\mathrm{Cr}$ & 0,02 & 0,098 & BLD & 0,005 & BLD & 0,003 & 0,001 \\
\hline $\mathrm{Mn}$ & 0,013 & 0,006 & BLD & BLD & 0,106 & 0,006 & BLD \\
\hline $\mathrm{Fe}$ & BLD & 1,227 & BLD & BLD & 2,094 & 0,55 & BLD \\
\hline $\mathrm{Ni}$ & 0,027 & 0,38 & BLD & 0,013 & BLD & BLD & BLD \\
\hline $\mathrm{Cu}$ & BLD & 0,491 & 0,026 & 7,469 & 0,014 & 0,017 & 0,007 \\
\hline $\mathrm{Zn}$ & BLD & BLD & BLD & 7,206 & 0,239 & 0,078 & 0,423 \\
\hline As & BLD & BLD & BLD & BLD & 1,918 & BLD & BLD \\
\hline $\mathrm{Se}$ & BLD & BLD & BLD & 0,013 & BLD & BLD & BLD \\
\hline $\mathrm{Cd}$ & BLD & BLD & BLD & 0,067 & BLD & BLD & BLD \\
\hline $\mathrm{Sb}$ & BLD & BLD & BLD & SLD & 0,062 & BLD & BLD \\
\hline $\mathrm{Pb}$ & 0,001 & 0,05 & 0,005 & 4,708 & 0,03 & 0,005 & BLD \\
\hline
\end{tabular}

\section{BLD: bajo el límite de detección}

Es interesante señalar que el análisis de dos filtros provenientes de un horno de cocción tomados con una diferencia aproximada de dos horas, mostró un cambio en las emisiones relativas de algunos elementos en dichas oportunidades La Figura 4 muestra una variación significativa de las concentraciones de $\mathrm{Cr}, \mathrm{Cu}, \mathrm{Mn}$, y $\mathrm{Zn}$, las que pueden tener su origen en diferencias en las condiciones operacionales y en el caudal de los gases de escape.

Puesto que el $95 \%$ de las partículas emitidas por el horno de cocción están en un rango de tamaño de partícula de 0,28 a $0,43 \mu \mathrm{m}$, dichos elementos se encontrarán en partículas muy pequeñas, que podrán alcanzar fácilmente los alvéolos pulmonares.

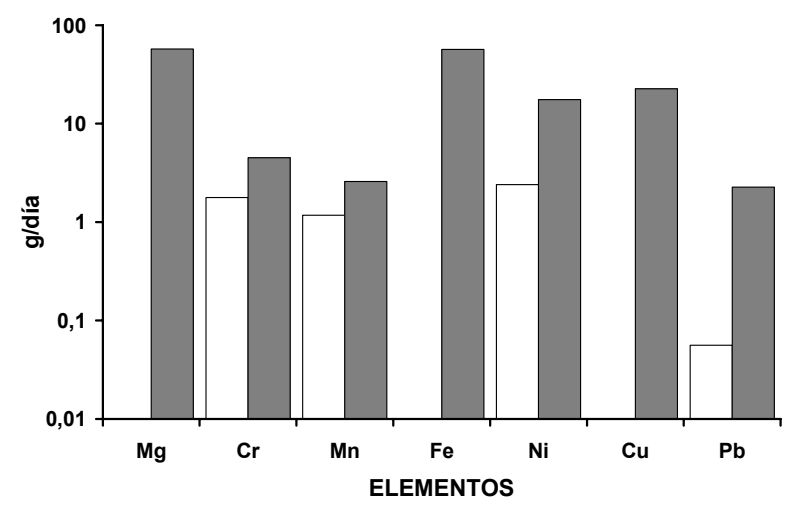

Fig. 3. Elementos cuantificados en las emisiones de dos calderas industriales.

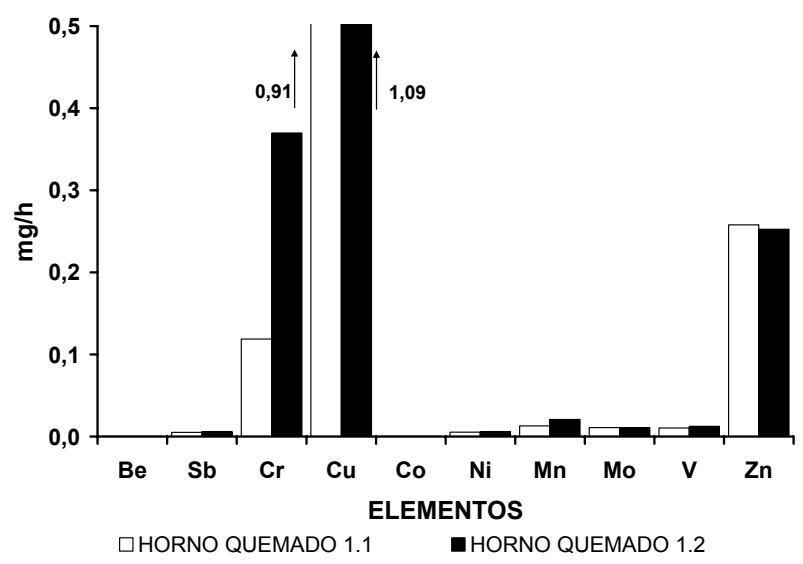

Fig. 4. Emisiones másicas-horarias de elementos para una misma fuente, en dos momentos del día.

La figura 5 muestra una comparación entre las emisiones diarias de elementos químicos encontradas en los filtros de un Horno Morgan y un Horno de Polimerización. Se detectaron diferencias en la cantidad de emisiones de $\mathrm{Cu}$ y $\mathrm{Pb}$ en ambos filtros. Por otra parte, $\mathrm{Cd}, \mathrm{Cr}$, $\mathrm{Ni}$, Se y $\mathrm{Zn}$, se detectaron sólo en el Horno Morgan.

La figura 6 muestra los resultados de las concentraciones elementales en emisiones de dos tipos muy diferentes de fuentes: un molino y un secador. En estas fuentes se detectó presencia de $\mathrm{Cu}, \mathrm{Fe}, \mathrm{Mn}, \mathrm{Pb}$ y $\mathrm{Zn}$ en ambas fuentes. As y Sb están presentes sólo en las emisiones del secador y $\mathrm{Cr}$ y $\mathrm{Mg}$ sólo en las del molino. 


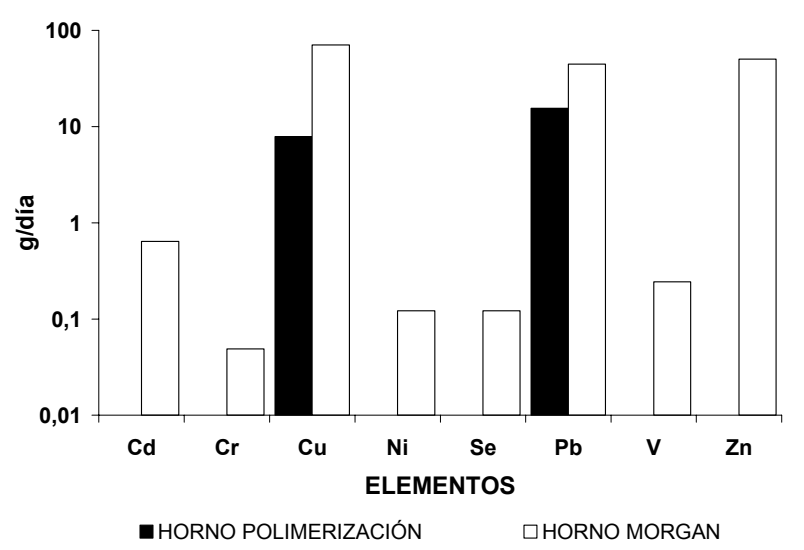

Fig. 5. Emisiones másicas diarias desde hornos de Polimerización y Morgan

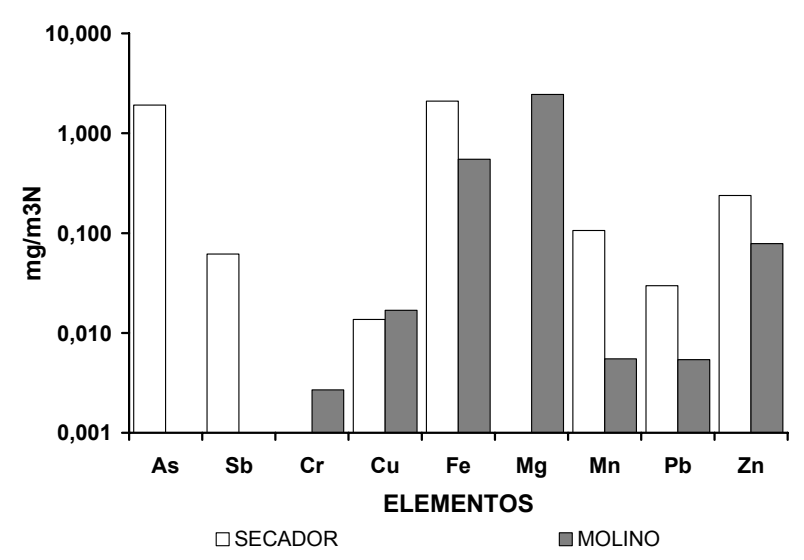

Fig. 6: Concentraciones elementales en las emisiones de un molino y un secador.

Cabe destacar que el secador, con una mayor variedad de elementos, tiene el máximo porcentaje de partículas en el rango más fino de 0,28 a 0,43 $\mu \mathrm{m}$. Las emisiones de $\mathrm{Fe}$ y $\mathrm{Zn}$ no son muy diferentes en ambas fuentes, sin embargo las emisiones de $\mathrm{Mn}$ y $\mathrm{Pb}$ son mayores en el secador y las emisiones de $\mathrm{Cu}$ son mayores en el molino.

Simulación del Proceso de Compensación de Emisiones

Con el objeto de simular y analizar una aplicación del sistema de compensación de emisiones antes descrito, se diseñó un escenario que considera un molino como fuente nueva que debe compensar sus emisiones con una pulidora existente. Los datos para ambas fuentes fueron extraídos de la base oficial de datos de fuentes estacionarias:

$\mathrm{C}_{\text {MOLINO }}=20,8 \mathrm{mg} / \mathrm{m}^{3}$

$\mathrm{C}_{\text {PULIDORA }}=2,7 \mathrm{mg} / \mathrm{m}^{3}$

$\mathrm{Q}_{\text {MOLINO }}=6,92 \mathrm{~m}^{3} / \mathrm{h}$
$Q_{\text {PULIDORA }}=19,9 \mathrm{~m}^{3} / \mathrm{h}$

$\mathrm{H}_{\text {MOLINO}}=24 \mathrm{~h} /$ día

H $_{\text {PULIDORA }}=24$ h/día

Aplicando la ecuación (1) se obtuvieron las siguientes emisiones diarias base para cada fuente (ecuaciones (2) y (3)):

$$
\begin{aligned}
& E_{D, \text { MOLINO }}=6,92 \cdot 208 \cdot 10^{-7} \cdot 24=3,5 \frac{\mathrm{Kg}}{\mathrm{dí} a} \\
& E_{D, \text { PULIDORA }}=19,903 \cdot 27 \cdot 10^{-7} \cdot 24=1,3 \frac{\mathrm{Kg}}{\text { día }}
\end{aligned}
$$

De acuerdo con la legislación chilena actualmente vigente, la nueva fuente debe compensar el $150 \%$ de sus emisiones, con lo que la cantidad a compensar resulta ser de $3,5^{\star} 1,5=5,3 \mathrm{Kg}$ día $^{-1}$. Por otra parte, la cuota de emisión para la fuente existente (pulidora), aplicando el estándar de $32 \mathrm{mg} \mathrm{m}^{-3}$ vigente desde diciembre del 2004, se muestra en las ecuación (4):

$$
\begin{aligned}
& E_{\text {СUОтА }}=19903 \frac{\mathrm{m}^{3}}{\mathrm{~h}} \cdot 32 \cdot 10^{-6} \frac{\mathrm{Kg}}{\mathrm{m}^{3}} \cdot 24 \frac{\mathrm{h}}{\text { día }} \\
& E=15,3 \frac{\mathrm{Kg}}{\text { día }}
\end{aligned}
$$

En consecuencia, dicha fuente tiene un cupo de emisión para ofrecer al mercado de casi 14 $K g$ día ${ }^{-1}$, permitiendo la entrada en operación de la nueva fuente con menor emisión $(5,3 \mathrm{Kg}$ día ${ }^{-1}$.

En términos prácticos, lo anterior implica un aumento neto de emisiones de material particulado de $3,5 \mathrm{Kg}$ día $^{-1}$, agregando cuatro elementos químicos ( $\mathrm{Fe}, \mathrm{Mg}, \mathrm{Mn}$ y $\mathrm{Pb}$ ) en las cantidades indicadas en la Tabla 4 a la vez que reduciendo las emisiones de $\mathrm{Cr}, \mathrm{Cu}$ y $\mathrm{Zn}$, como se indica en la misma Tabla.

Tabla 4: Emisiones anuales de elementos desde un molino y una pulidora ( $K g$ año $\left.{ }^{-1}\right)$

\begin{tabular}{crr}
\hline Elemento & \multicolumn{1}{c}{ Molino } & \multicolumn{1}{c}{ Pulidora } \\
\hline $\mathrm{Cr}$ & 0,8 & 3,2 \\
$\mathrm{Cu}$ & 5,2 & 27,0 \\
$\mathrm{Fe}$ & 168,0 & --- \\
$\mathrm{Mg}$ & 750,0 & -- \\
$\mathrm{Mn}$ & 1,7 & --- \\
$\mathrm{Pb}$ & 1,7 & --- \\
$\mathrm{Zn}$ & 24,0 & 1769,0 \\
\hline
\end{tabular}

Información Tecnológica - Vol. 18 No 2 - 2007 


\section{DISCUSIÓN}

El sistema de compensación de emisiones es una herramienta de gestión ambiental pública incluida en el PPDA-RM, que ha sido aplicada principalmente a calderas con caudal volumétrico mayor que $1000 \mathrm{~m}^{3} \mathrm{~h}^{-1}$. Su aplicación a hornos y procesos industriales es más compleja, dado que la ecuación para calcular la cuota de emisión no es tan directa como en el caso de calderas.

El principal objetivo de esta herramienta de gestión es reducir, o al menos congelar, las emisiones totales de material particulado en la región metropolitana de Santiago, estimulando la incorporación de nuevas tecnologías así como también la conversión desde combustibles fósiles convencionales a combustibles más limpios como gas natural y electricidad. Mediante este sistema, fueron asignadas cuotas o "derechos" de emisión a las fuentes existentes y las nuevas fuentes o expansiones de las existentes quedaron con una cuota nula de emisión, debiendo, además, compensar emisiones con reducción de emisiones de fuentes existentes.

Cuando se aplica el sistema de compensación a emisiones gaseosas, se verifica una compensación entre compuestos de composición química análoga, no siendo éste el caso del material particulado. Los resultados de este estudio muestran que las emisiones de material particulado son de naturaleza muy compleja e, incluso en el caso de la compensación de emisiones entre fuentes del mismo tipo, pueden darse notables diferencias en el tipo y cantidad de elementos químicos específicos, así como en la distribución del número de partículas por tamaño, lo que se traduce en diferentes efectos sobre la salud humana.

Si bien en este estudio se analizaron muestras de una reducida cantidad de fuentes, las muestras seleccionadas cubren un amplio espectro de tipos de fuentes, incluyendo no solo calderas, sino que también diferentes tipos de hornos y procesos industriales. El reducido número de filtros analizados químicamente se debe a que las bases de datos oficiales de fuentes estacionaras no tienen información completa, lo que no permite calcular las concentraciones de elementos en todos los filtros disponibles.
En el período 1997-2000, se ha podido cuantificar una notable reducción de las emisiones de material particulado desde fuentes estacionarias en la Región Metropolitana de Santiago (Corvalán et al, 2003), y varias fuentes emisoras existentes cumplen con el estándar de $32 \mathrm{mg} \mathrm{m}^{-3}$, que se utiliza para definir las cuotas de emisión desde diciembre del 2004. Este hecho implica un enorme potencial de compensación de emisiones de nuevas fuentes, lo que podría significar un aumento neto de emisiones, como lo demuestran los resultados de este estudio.

Dado el incremento de las emisiones totales de material particulado, las compensaciones que eventualmente tengan lugar entre fuentes que emiten material particulado de diferente composición química y distribución en tamaño de partícula, pueden hacer que la calidad del aire aumente su grado de deterioro.

Hay, por otra parte, otros factores no considerados en este estudio, como la localización geográfica de las fuentes y la dirección del viento que pueden influir significativamente sobre los efectos en la salud de la población que vive en las zonas de influencia de las fuentes involucradas, aspectos no considerados en el diseño del sistema de compensación.

\section{CONCLUSIONES}

Los resultados de este estudio permiten concluir que el sistema de compensación de emisiones actualmente utilizado como herramienta de gestión ambiental pública para reducir o al menos congelar las emisiones de material particulado, y con ello mejorar la calidad del aire, sobre la base de las emisiones másicas totales, puede ser ineficiente para los propósitos señalados.

Adicionalmente a las emisiones másicas totales, es necesario incluir análisis físicos y químicos, esto es, considerar la composición en elementos químicos de las emisiones de las fuentes y focalizarse en las partículas $100 \%$ respirables, que corresponden, al menos, al $90 \%$ del total de partículas captadas por los filtros, de acuerdo con lo demostrado en este estudio.

Dada la caracterización individual de cada fuente, en relación con su caudal másico, tipo 
de proceso y características físicas y químicas de los combustibles, entre otras variables, el conocimiento y comprensión de la composición química y la distribución de tamaño de las partículas son de la mayor importancia.

Es además posible concluir que una fuente puede presentar variaciones en la concentración elemental relativa a lo largo del día. Esto puede ser el resultado de variaciones del flujo de gases de escape de la fuente que provienen de diferentes condiciones operacionales.

Para incorporar la información que se desprende de esta investigación básica en el sistema de compensación de emisiones, es necesario definir grupos de fuentes con emisiones homogéneas de material particulado, en términos de distribución de tamaño de partícula y composición química. Esto permitiría definir cuotas de emisión ponderadas según estos dos parámetros.

Con el objeto de lograr lo recomendado anteriormente es necesario llevar a cabo un programa experimental de caracterización física y química de material particulado utilizando una muestra bastante mayor que la utilizada en la presente investigación y con un mayor grado de representatividad de las fuentes y procesos industriales presentes en la región.

\section{AGRADECIMIENTOS}

Agradecemos a: la Autoridad Sanitaria de la Región Metropolitana, por los filtros utilizados en esta investigación, a los Químicos Leonor Garay, Carlos Ferrada y Rubén Verdugo por los análisis físicos y químicos y al proyecto TNAC-05/03 de la Universidad de Chile por el financiamiento de esta investigación.

\section{REFERENCIAS}

Baik, N., Kim, Y. y Moon, C., Visibility Study of Seoul. Atmospheric Environment, 30(13), 2319-2328. (1996).

Carter, J. D., Ghio, A. J., Samet, J. M. y Devlin, R. B., Cytokine Production by Human Airway Epithelial Cells after Exposure to an Air Pollution Particle Is Metal-Dependent. Toxicol. Appl. Pharmacol, 146(2), 180-188. (1997).
Cohen, B. y Hering, S., Air Sampling Instrument for Evaluation of Atmospheric Contaminants. American Conference of Governmental Industrial Hygienists. Cincinnati, Ohio, 8th ed. (1995).

Corvalán, R.M., Galecio, J.I y Salim, J.A., Effectiveness in the Use of Natural Gas for the Reduction of Atmospheric Emissions: Case Study-Industrial Sector in the Metropolitan Region of Santiago, Chile. J. of the Air \& Waste Management Assoc., 53, 971-975. (2003).

Costa, D. L. y Dreher, K. L Bioavailable transition metals in particulate matter mediate cardiopulmonary injury in healthy and compromised animal models. Environ Health Perspect., 105(suppl 5):1053-1060. (1997).

Donaldson, K., Li, X. Y. y MacNee, W., Ultrafine (Nanometer) Particle Mediated Lung Injury. J. Aerosol Sci., 29, 553 - 560. (1998).

Duffus, J. H., Toxicología Ambiental. España: Editorial Omega. (1983).

Goyer, R., Nutrition and Metal Toxicity. Am. J. Clin. Nutr., 61, 646S-650S. (1995).

Hosiokangas, J., Ruuskanen, J. y Pekkanen, J., Effects of Soils Dust Episodes and Mixed Fuel Sources on Source Apportionment of PM 10 Particles in Kuopio, Finland. Atmospheric Environment; 33(23), 821-830. (1999).

Lazar, A., Reilly, P. y Whitten, W., Real-time Surface Analysis of Individual Airborne Environment Particles. Environmental Science \& Technology, 33(22), 3993-4001. (1999).

MINSAL. Diario Oficial de la República de Chile. Decreto Supremo $N^{\circ} 4$. Ministerio de Salud. 02 de Marzo 1992. (1992).

Morawska, L., Thomas, S., Jamriskja, M. y Johnson, G., Modality of Particle Size Distribution of Environmental Aerosols. Atmospheric Environment. 33(27), 44014411. (1999).

MSGP República de Chile. Ministerio Secretaría General de la Presidencia. Decreto Supremo $N^{\circ} 20$. Modifica al D.S. No 16/1998. (www.conama.cl/portal/1255/articles25999_pp da.pdf). (2003). 
Mukherjee, A.B., Káántee, U. y Zevenhoven, R., The Effects of Switching From Coal to Alternative Fuels on Heavy Metals Emissions from Cement Manufacturing. In: Chemistry of trace elements in fly ash, eds.: K.S. Saiwan, A.K. Alva \& R.F. Keefer, pp. 45-61.New York: Kluwer Academic. (2003).

Ntziachristos, L. y Samara, Z., New Directions: Emerging demands for vehicle particle emission characterization. Atmospheric Environment, 37(3); 299-442. (2003).

Ondov, J.M. y Wexler, A.S., Where Do Particulate Toxins Reside? An Improved Paradigm for the Structure and Dynamics of the Urban Mid-Atlantic Aerosol. Environ. Sci. Technol., 32, 2547-2555. (1998).

Phalen, R. F., The particulate air pollution controversy: a case study and lessons learned. $\quad 2^{\text {nd }}$ printing. Kluwer Academic Publishers. (2004).

Préndez; M., Ortiz, J., Zolezzi, S., Campos, C. y Apablaza, N., Aerosoles Atmosféricos de Naturaleza Inorgánica. Contaminación en Santiago de Chile. Revista Chilena Enferm. Respir., 7(4), 224-237. (1991).

Préndez, M., Características de los Contaminantes Atmosféricos. En Contaminación Atmosférica de Santiago, Estado Actual y Soluciones, eds. H. Sandoval, M.
Préndez y P. Ulriksen, pp.111-186. Cabo de Hornos, Santiago, Chile. (1993).

Sienra, M. R., Rosazza, N.G. y Préndez, M. M. Polycyclic aromatic hydrocarbons and their molecular diagnostic ratios in urban atmospheric respirable particulate matter. Atmospheric Research 75, 267-281. (2005).

Sosaky, K., Kurita, H., Carmichael, G., Chang, G. y Muran, K., Behavior of Sulfates, Nitrates and other Pollutants in the Long-range Transport of Air Pollution. Atmospheric Environment, 22(7), 1301-1308. (1988).

Sudesh Y. and Rajamani V. Air quality and trace metal chemistry of different size fractions of aerosols in N-NW Indiaimplications for source diversity. Atmospheric Environment, 40(4), 698-712. (2006).

US EPA Emisiones de Tóxicos en el Aire: Estrategia de la EPA para reducir Riesgos en Salud en Áreas Urbanas. Oficina de Calidad de Aire, planificación y estándares (1999).

Vana, M., Tamm, E. y Vill, M., Experimental Study of the Air Pollution Transport by Synchronized Monitoring of Atmospheric Aerosols. Atmospheric Environment, 33(28), 4615-4628. (1999).

WHO Air Quality Guidelines for Europe $2^{\text {nd }}$ ed. WHO Regional Publication European Series, No91, Copenhagen (2000). 